tigungen unseres Landes, welche zugleich $\mathrm{zu}$ den bedeutendsten Einkünften des Staates gehört.

Nicht so gut gedeihet eine zwei Stunden lange Allee mit Populus alba, welche nach dem Pyräus führt, denn sie hat während der Sommermonate eine solche Hitze zu überstehen, dass alljährlich Hunderte von Bäumen vertrocknen und dann ausgerottet werden müssen. Auch eine Alle von Ulmen an der Strasse vom königlichen Palais bis zur Universität und von da bis zur Strasse nach Patysia will nicht gut gedeihen. Erst erproben muss sich auch eine Allee, die vor wenigen Monaten auf dem Otto-Platze mit Ceratonia Siliqua und Nerium Oleander angelegt wurde.- Im Falle diese Bäume fortkommen sollten, wird auch die Anlage in wenigen Jahren ein prachtvolles Ansehen darbieten.

Ueberhaupt ist die Erhaltung der sämmtlichen Alleen von Athen mit vielen Schwierigkeiten verbunden, hauptsächlich aber wegen des Nangels an Wasser, dass allen diesen Bäumen während der Sommermonate zugeführt werden muss. *)

So gestaltet sich Athen, einst die Stadt der Wissenschaft und Künste und dann für lange dem Verfalle überlassen, nun wieder von Tag zu Tag schöner; Wissenschaft und Künste erheben sich wieder an Minerven's einstiger Stätte und es regt sich allenthalben eine bewunderungswürdige physische und moralische Thätigkeit, hervorgerufen, angeeifert und geschützt von Griechenlands Genius, unserer gefeierten Königin Amalie!

Athen, im Jänner 1861.

\title{
Correspondenz.
}

Brandenburg, a. d. Havel, im Mai 1861.

Von der dritten Jahresversammlung des bot. Vereins für die Provinz Brandenburg und die angrenzenden Länder so eben zurückgekehrt, erlaube ich mir, Ihnen Einiges von dem frohen Feste, das wir nun zum dritten Male zusammen verlebt, mitzutheilen. Unser Verein wurde, wie auch Ihr geschätztes Blatt berichtete, vor zwei Jahren in Neustadt-Eberswalde gegründet. Vorstand desselben sind die Herren Prof. Dr. Alexander Braun, Prof. Dr. Ratzeburg, Dr. Paul Ascherson (Schriftführer), Dr. Liebe, Whajor a. D. von Jasmund. Zweck des Vereines ist "das Studium der Botanik auf seinem Gebiete - die Provinz Brandenburg und der ebene Theil der Provinz Sachsen, so wie die Anhaltischen Herzogthümer - besonders aber die Erforschung seiner Flora zu befördern." Er besteht bereits aus mehr als 120 Mitgliedern und

*) In Wien nahm man es bis jetzt mit den Bäumen auf dem Glacis nicht so genau. daher kam es auch, dass in warmen Jahren schon zu Ende Juni das Laub verdorrt von den Bäumen hing.

Anm. d. Redact. 
hat seine "Verhandlungen" in zwei Heften pro 1859 und 60 veröffentlicht. Letztere geben das anschaulichste Bild von der rüstigen Thätigkeit der Mitglieder, deren Zahl fort und fort wächst, da ohnehin nur der niedrige Jahresbeitrag von einem Thaler Einzelnen wie ganzen Gesellschaften den Eintritt erleichlert. Möchten auch diese Zeilen dazu beitragen, ihm aus der Ferne her neue Freunde zuzulühren! *) - Unsere Jahresversammlungen finden an jedem Dinstag nach Pfingsten Statt und zwar stets an andern Orten der Mark, so die begründende in Neustadt-Eberswalde, die vorjährige in Potsdam, die heurige in Nauen. Für den Versammlungsort pro 1862 ist Frankfurt a. d. Oder bestimmt worden und zugleich eine Excursion durch die anmuthige Gegend von Buckow verabredet worden. Prof. Dr. Brau n eröffnete die diesjährige Sitzung, die in einem Gasthofe an der Eisenbahn abgehalten wurde, mit einem Gruss und einer kurzen Ansprache an die Versammlung, worauf der Secretär, Dr. P. Ascherson, den Jahresbericht vortrug und das übrige Geschäftliche abgethan wurde. Einem Mitgliede war die Gesellschaft zu besonderem Danke verpflichtet. Herr Schw einfurth hatte zum zweiten Hefte der Verhandlungen mit kunstgeübter Hand die nöthigen Abbildungen gezeichnet, lithographirt und zum Geschenk gemacht, wofür ihm von der Gesellschaft durch Aufstehen der wohlverdiente Dank dargebracht wurde. Der erste Vortrag des Herrn Prof. Schultz-Schultzenstein verbreitete sich über Standorte gewisser Pflanzen und ich theile Ihnen darüber Einiges nachstehend mit. Leersia oryzoides $\mathrm{S}_{\mathrm{w}}$, eine in der Mark z. B. am Rummelsburger See nicht eben häufige Pflanze, findet sich bei Rheinsberg und Zechlin massenhaft, wo sie Quellen und kaltgründigen Boden liebt. Sie blüht aber später, als sonst in den Floren angegeben wird, nämlich im October und Anfang November. Arnica montana L. steht bei Ruppin vereinzelt, an den Havelufern von Zehdenik, in dem sogenannten "Hundebusch" auf mit Rasen überzogenem, nicht nassen Boden in Menge für den Apothekenbedarf. Hierzu erinnert Schramm an die Standorte bei Rathenow, Genthin und Bredow, Dr. Ascherson an Landin, und die Herren Grantzow und Buchholz bemerken, dass die Landleute unter dem deutschen Namen der Arnica "WohIverley" auch Imula britannica und Authyllis vulneraria zu medicinischen Zwecken gebrauchen. Pulmonaria officinalis $L$. ist vom Vortragenden vereinzelt beim Pfefferteich in der

*) $\$$. 3 der Statuten: ,Ordentliches Mitglied ist Jeder, der die Zwecke des Vereines durch einen Jdhresbeitrag von einem Thaler pr. Cour. befördert, “ - \$. 6. Der Verein veröffentlicht seine Verhandlungen in zwanglosen Heften, eventuell jährlich. Dieselben enthalten den Bericht über die Versammlungen, so wio wissenschaftliche Aufsätze und Correspondenzenüber Gegenstände der Vereinsthätigkeit. Jedes Mitglied erhält dieselben unentgeltlich." - Erstes Heft mit Beiträgen von A s cherson, Bolle, Braun, Irmisch, v. Klinggraf, Paukert, Ratzeburg, Reinhardt. Nit 2, Steindru ktafelı, ungefähr 7 Bogen stark. - Zweites Heft mit Beiträgen von 20 Nityliedern. XXII und über 14 Bogen nebst 3 Tafeln. 
Neu-Ruppiner-Gegend auf sogenannten rauhen Stellen gesammelt worden und zwar in einer Niederung von Buchen und Eichen. In Lanke wird es dagegen kiepenweis zu Markte gebracht. - Dr. Ascherson: Auf unserem Gebiete kommt es nie gefleckt vor, wie z. B. bei Posen. Crepis praemorsa Tausch (Hierac. praem. L.s) steht im Tornowsee auf einer kalkhaltigen Insel mit Salvia pratensis und Ophrys arachnites, welche letzlere leider im letzten Jahre ausgeblieben ist. Ebenso steht sie bei Karlswerk. Scirpus Tabernaemontani Gm. wird im Ruppin'schen zu Bündeln zusammengebunden, an welche man Angelhaken befestigt, um Aale zu fangen ("Aalflossen"). Scirpus lacustris L. lässt sich dazu nicht verwenden, letztere ist zu leicht, wird bald vom Bindfaden durchschnitten; erstere wird desshalb von den Fischern auch "Steinbinse" genannt und der Vortragende glaubt, dass diese Härte einen guien Unterschied zu den wenigen, welche die beiden Arten trennen, noch abgeben könne. Ueber den Standort von Sc. Tab. sind nicht alle Herren gleicher Meinung, der eine hat sie mehr an feuchten, ausgetrockneten Gräben, der andere auf kiesigem Boden in einem kleinen See beobachtet. Linnaea borealis Gr. Bei Berlin finden sich drei Standorte dieser schönen Pflanze, ein anderer in Mecklenburg, in der Ruppiner Stadtforst "Hakspitze" steht sie sogar in ziemlich grosser Menge. Sie ist aber gegen kaum etwas veränderten Boden beim Verpflanzen sehr empfindlich und geht leicht wieder aus. In Schweden findet sie sich nicht unter Moos, sondern auf nacktem Boden, der thonig, mergelig oder glimmerig ist; in botanischen Gärten daselbst aber auch auf hellen, nicht schattigen Orten cultivirt. Herr Oekonomierath $\mathrm{Sch} \mathbf{r a m m}$ hat sie in dem Forst Grünane bei Rathenow auf Moos mit Pteris aquilina beobachtet, in Graubündten aber zwischen Felsen und Steingeröll. Dr. Ascherson hält sie nur für wählig mit Rücksicht auf Verpflanzung an den Grenzen ihres Verbreitungsbezirkes. Dass sie blos nach kalten Wintern bei uns zum vollkommenen Blühen komme, was der verstorbene Prof. Link behauptet, wird angezweifelt. Hieran knüpfte der Redner noch einen Wunsch, betreffend die Nomenclatur der märkischen Pflanzen. Er bat die Herren Mitglieder, sich der älteren von den bekannten märkischen Floristen Willd enow, Kunth u. s. w. gebrauchten Namen zu'bedienen und die neueren Veränderungen und Umtauschungen in den Namen der Gattungen und Arten abzulehnen, und dies, wie er sich ausdrückte, aus Patriotismus. Dagegen trat Dr. P. As cherson auf, wollte die Priorität der Namengebung auf's Strengste beobachtet wissen und machte geltend, dass wie wir, so auch alle Länder und Ländchen das Recht hätten, aus Patriotismus ihren Floristen zu folgen. Er schloss mit den Worten: „Es gibt keine märkische, schlesische oder irgendwie provincielle Botanik, sondern nur eine einige, wissenschaftliche." Vermittelnd trat Prof. Alex. Braun zwischen beide Ansichten. Rücksichtlich der Prioritäten in der Namengebung müsse es doch eine Grenze geben, über dic hinaus zu gehen, nicht immer räthlich und thunlich sei. Möglicher- 
weise dürfe man dann auch noch vor Linn és Zeit Prioritäten verfolgen, man thäte daher besser, um zu einem Ende zu kommen, man nähme an, dass auch solche Anrechte verjähren könnten. Gegen gewisse Aenderungen dagegen könne man sich nicht verschliessen. Wenn ein früherer Name offenbare Unrichtigkeiten enthielte, wie beispielsweise Lunaria annua, die doch biennis sei, so müsse man der Neuerung folgen. Andere Pflanzen, denen erst ein enger Verbreitungsbezirk im Beinamen zugeschrieben wurde, der sich später als bei Weitem unzureichend herausstellte (Ajuga Genevensis z. B.) möchten immerhin ihren Namen behalten. Wenn endlich eine Confusion zwischen mehreren Arten Statt gefunden habe und man sich nicht mehr verstehe, so bleibe nichts weiter übrig, um einer gänzlichen Sinnverwirrung vorzubeugen, als das einfache Annehmen der neuen Bezeichnung. Herr Dr. Behncke zeigte darauf einen Granatapfel vor, der im Schöneberger botanischen Garten gereift war und sehr auffallende Haarbüschel einer Echinocactusart, welche er aus Mexico erhalten und welche die grösste Aehnlichkeit mit thierischer Wolle hatten. Herr Oekonomierath Schramm vertheilte darauf verschiedene Exemplare der eben im Druck vollendeten Nachträge zu seiner Flora der Stadt Brandenburg und Umgegend und Herr Actuar Schultze aus Königshorst bei Nauen theilte eine Anzahl frischer Exemplare des bei uns seltenen Allium ursinum L. mit, die reichlich Abnehmer fanden. Hierauf begann der mehrfach schon genannte Vorsitzende, Herr Prof. Braun, einen längeren Vortrag morphologischen Iahalts über abnorme Blattbildungen, welcher von einem umfangreichen Blattherbar unterstützt wurde. Mit gespanntester Aufmerksamkeit verfolgten die Hörer denselben, sie hatten vielfache Gelegenheit, die verschiedenarligsten Gestaltungen des Blattes zu bewundern, von der grössten Ausbreitung der Blattfläche an bis zur höchst möglichsten Contraction, die sich zuletzt als einfache Mittelrippe darstellt, nebst einer sehr grossen Zahl charakteristischer Uebergange. Da sich die Erklärungen des Vortragenden ganz an das Herbar anschlossen, ist es schwer, etwas davon wiederzugeben. Um jedoch Eins hervorzuheben, da von Tutenbildungen an verschiedenen Blättern in botan. Zeitungen mehrfach in neuerer Zeit die Rede gewesen ist, so sei es mir erlaubt, auf die Beispiele hinzuweisen, an welche sich dieser Theil des Vortrags gerade anschloss. Es waren folgende: Blätter mit Tutenbildung von Tilia, Ulmus (hier zeigte sich der Blattstiel erst verlängert, ehe er sich in die Tute ausbreitete, Populus canescens, bei Corylus häufig, Pelargonium, Pisum sativum, Acroglochin, Saxifraga, Euphorbia, Rosa, Potentilla, Staphylea, ein fünfblättriges Exemplar von Trifolium pratense mit der Tutenbildung Glycirrhiza foetida, Gleditschia triacanthos sehr häufig, Rhus typhina. Genähte Blätter fanden sich an Aristolochia, Morus alba, Corylus Avellana, Gesneria, Ceratonia Siliqua, ferner Emergenzen an Saxifraga crassifolia u. $\mathrm{s}$. w. Ein heiteres Mahl folgte darauf und der Nachmittag ward einer Excursion gewidmet, welche diesmal 
die bekannten Salzwiesen zu beiden Seiten des Dechtower Dammes und einen Theil der Stadtforst von Nauen, Apfelhorst genannt, zum Ziele hatte. Leider war die Vegetation durch die vorangegangenen kalten Tage sèhr aufgehalten worden. Vieles, was wir suchten und erwarten durften, war zum Theil noch ganz unentwickelt, so Glaux maritima L. und Melilotus dentata Pers. Ich beschränke mich desshalb, nur anzuführen, dass in dem stehenden Wasser längs des Dammes neben verschiedenen Charen Nitella glomerata und das unserm vaterländischen Botaniker und Arzte, dem alten Doctor Heim, zu Ehren benannte Laubmoos Pottia Heimii eingesammelt wurden. - Der nahende Abend endlich zerstreute die heitere Gesellschaft wieder; die Eisenbahnzüge entführten die Gäste, welche zum Theil aus weiter Ferne gekommen waren, sämmtlich dankbar für das schöne Wetter und die gehabten Genüsse. W. Hechel.

\section{Flora austriaca.}

Hypnum fallaciosum $\mathrm{n}$. sp. (Juratzka in den Verhandlungen der zool.-bot. Gesellschaft vom 1. Mai 1861.) Laxe caespitans, caulis procumbens et ascendens parce radiculosus vage ramosus; rami flaccidi vage vel subpinnatim ramulosi, ramulis erecto-patentibus, folia ramea remotiuscula, ramulina confertiora modice squarroso-patula, apicalia saepius subfalcato-secunda, ex ovata vel cordato-ovata basi lanceolata longe acuminata margine integra, subplana, mollia; costa bifurca crure altero brevi, altero longiori haud raro ad medium producto, areolatione (illae Hypni Kneiffi simili) peranguste rhomboideo-hexagona basi laxiore, ad angulos excavatos inflato-dilatata. Flores polygami, masculi antheridiis 6-12 longe paraphysatis, hermaphroditi antheridiis et archegoniis paucis, feminei angustiores archegoniis numerosis; perichaetium basi radiculosum foliis inferioribus ex ovato subito anguste acuminatis e medio patulis subecostatis, superioribus late lanceolatis subito fere longe tenuique acuminatis, plicato-sulcatis tenui-costatis. Capsula in pedicello elongato flexuoso e basi erecta incurvo-cernua operculo convexo-conico apiculato, annulo lăto. Peristomii dentes superne late hyalino-marginati, processibus integris, ciliisque ternatis exapendiculatis. Fruct. matur. aestate. Habitat locis humidiusculis ad Danubium prope Vindobonam ; in "Radegger Moor" prope Juvaviam (Fr. Bartsch); prope Vratislaviam Silesiae (Dr. Milde) *et prope Senftenberg Bohemiae orientalis (Em. Weiss).

\section{Personalnotizen.}

- Dr. Theodor Kots chy, Custos-Adjunct am botanischen Hofkabinet wurde, von der kaiserl. Akademie der Wissenschaften zu ihrem correspondirenden Mitgliede gewählt. 